Conclusions Dermal lymphatic network of lupus models have never been characterized, despite evidence for a major role of lymphatics in the regulation of chronic inflammation and autoimmunity. Here we provide indications to impaired long-term response of lymphatic drainage in the lpr lupus strain, which can lead to reduced lymphatic regulation of the immune response, contributing to the unchecked skin inflammation known to occur in SLE.

Funding Source(s): NIH T32AR071302-01 (NS)

NIH R01AI079178 (TTL)

Lupus Research Alliance (TTL)

\section{IMPACT OF THE DIAGNOSIS OF JUVENILE SYSTEMIC LUPUS ERYTHEMATOSUS IN PATIENTS AND THEIR PARENTS}

${ }^{1}$ Maria Carolina dos Santos*, ${ }^{2}$ Milena Del Santo Rosa, ${ }^{1}$ Fabiola DA Kuchiki, ${ }^{1}$ Andressa GF Alves, 'Silvana B Sacchetti. ${ }^{1}$ Santa Casa de São Paulo; ${ }^{2}$ Hospital Geral de Guarulhos

10.1136/lupus-2019-Ism.110

Background The influence of psychosocial aspects on Juvenile Systemic Lupus Erythematosus (JSLE) is known both in the triggering of the disease and in reactivation. Since it is a chronic disease in pediatric patients, its diagnosis has an important impact on both the patient and the family. The understanding of the diagnosis, evolution and treatment of the disease leads to a better adherence to the treatment, consequently better evolution.Objective: To evaluate the impact of the diagnosis of JSLE in the life of patients and parents and the degree of understanding about the disease and treatment.

Methods Pilot study with application of a questionnaire containing epidemiological data, questions about the understanding of the disease, psychological impact on diagnosis and currently and association with a stressor event. This questionnaire was applied to the jSLE patients and their parents accompanied at pediatric rheumatology department of Santa Casa de São Paulo. Qualitative data were submitted to exploratory descriptive analysis.

Results 24 patients and 21 parents answered the questionnaire. $84 \%$ of the patients were female and $95 \%$ of the relatives were mothers. The age ranged from 9 to 17 and from 28 to 46 years for the patients and the parents, respectively. The follow-up time was $33.3 \pm 5.7$ months. Only $21 \%$ of the patients and $38 \%$ of the parents were able to define SLE as an autoimmune disease. Regarding the cause of SLE, $29 \%$ of parents and 13\% of children do not know but many parents associated with the emotional issue, while patients related to altered immune system. Both parents and patients associated a stressor event with the onset of jSLE. Regarding treatment both children and parents demonstrated an awareness of the need for appropriate medication and follow-up, but also described the importance of sun protection.

The main feeling reported at the time of diagnosis was sadness for both parents and patients. Already today, 50\% of children and parents express a feeling of conformity and tranquility, knowing how to deal better with the disease, but still have some degree of concern.
Conclusions jSLE is a disease that brings a sense of sadness and there is an association of a stressor event for both patients and parents. The health team must be able to clarify the lupus disease, considering limitations of understanding of the patients and parents, which allows a better control of the disease, bringing tranquility.

Funding Source(s): None

\section{KIKUCHI-FUJIMOTO DISEASE IN CHILDHOOD ONSET LUPUS: A CASE SERIES FROM A TERTIARY CARE CENTER IN NORTH INDIA}

${ }^{1}$ Himanshi Chaudhary*, ${ }^{2}$ Deepti Suri, ${ }^{3}$ Amit Rawat, ${ }^{3}$ Anju Gupta, ${ }^{2}$ Ankur Jindal, ${ }^{2}$ Sandesh Guleria, ${ }^{3}$ Bishan Radotra, ${ }^{2}$ Surjit Singh. ${ }^{1}$ Allergy Immunology Unit, Advanced Pediatrics Center, PGIMER Chandigarh; ${ }^{2}$ Postgraduate Institute of Medical Education and Research, Chandigarh, India; ${ }^{3} P G I M E R$ Chandigarh

\subsection{6/lupus-2019-Ism.111}

Background Kikuchi-Fujimoto disease (KFD) is a rare disorder described mostly in adolescents and young adults. It is a great mimicker, has a diverse clinic-laboratory profile and appears to be an under-recognized disease entity, especially in children. Methods We report 6 children who presented with fever and lymphadenopathy and had findings on fine needle aspiration cytology (FNAC) and/or histopathology that were compatible with a diagnosis of KFD. Two amongst these 6 patients developed lupus.

Results Case 1: A 7 year old boy was admitted with complaints of high-grade fever and left submandibular lymph node swelling for 2 weeks. A possibility of suppurative lymphadenitis was kept and he was treated with antimicrobials. He was the youngest born to a consanguineously married couple who had lost two children previously to a lupus-like illness. The striking family history suggested the possibility of a monogenic form of lupus (early complement deficiency). C1q levels were found to be significantly low (C1q level: $0.27 \mathrm{mg} / \mathrm{L}$; normal $(102-170 \mathrm{mg} / \mathrm{L}))$ Genetic analysis revealed a nonsense mutation in the C1QA gene (c.622C>T Q208X). Investigations revealed mild anemia (hemoglobin:107 gm/L) and elevated erythrocyte sedimentation rate (ESR:68 mm) in 1 st hour and C-reactive protein (CRP: $8 \mathrm{mg} / \mathrm{dl})$. An excision biopsy of the lymph node showed necrotizing histiocytic lymphadenitis consistent with KFD. Immunological work-up revealed Antinuclear antibodies (ANA) 2+speckled pattern on immunofluorescence; no anti ds-DNA antibodies; no antiphospholipid antibodies. He was treated with immunosuppressants and twice-daily fresh frozen plasma. Fever subsided in 5 days and lymph nodes regressed in 2 weeks.

Case 2: A 12-year-old boy presented with fever of 7 months duration and generalized seizures followed by altered sensorium. He was treated in lines of subacute meningo-encephalitis. As there was no response to treatment he was referred to our institute. On examination, he had malar rash, frontal alopecia and bilateral, multiple enlarged cervical lymph nodes. Laboratory investigations revealed anemia, elevated ESR, high CRP and low levels of C3 and C4. ANA was positive $(3+$ speckled); anti-dsDNA titers were $130 \mathrm{IU} / \mathrm{ml}$ (normal$<60 \mathrm{IU} / \mathrm{ml}$ ); FNAC from lymph node revealed features consistent with KFD. A diagnosis of lupus and KFD was made and he was started on oral prednisolone along with 


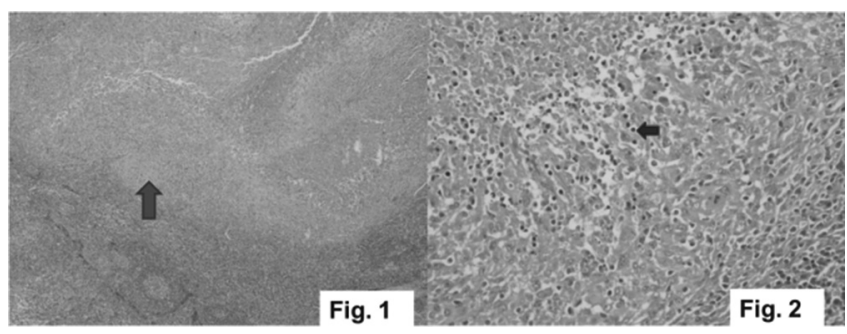

Abstract 111 Fig. 1 Histopathology of lymph node of KFD; areas of necrosis surrounded by histiocytes(red arrow). Fig. 2 The necrotic areas contain nuclear debris some of which is engulfed by histiocytes (blue arrow)

hydroxychloroquine. Fever subsided in 3 days and lymph nodes regressed in 2 weeks. There has been no recurrence of KFD on follow-up over the next 6 years.

Conclusions The association of KFD with childhood onset lupus is unusual. Early recognition of this entity can prevent diagnostic and therapeutic errors

Funding Source(s): nil.
Kikuchi-Fujimoto disease in childhood onset lupus: a case series from a tertiary care center in North India

\section{TRENDS IN TREATMENT OF SYSTEMIC LUPUS ERYTHEMATOSUS AND LUPUS NEPHRITIS: 2006-2016}

Ali Duarte-Garcia*, Cynthia Crowson, Chowdhary Vaidehi, Shreyasee Amin, Kenneth Warrington, Eric Matteson, Nilay Shah. Mayo Clinic

10.1136/lupus-2019-Ism.112

Background Glucocorticoids, anti-malarials and conventional immunosuppressive agents have been the mainstay of therapy for systemic lupus erythematosus (SLE) and lupus nephritis (LN); more recently biologic agents have been introduced for their treatment. We evaluated utilization trends in SLE and LN therapy over the past decade.

Methods Using 2006-2016 data from a large administrative database of commercially insured and Medicare Advantage beneficiaries, we identified patients with SLE and a subset of patients with LN based on validated claims-based algorithms. Included patients had at least one year of enrollment in the

Abstract 112 Table 1 Proportion of patients with SLE and LN receiving any of the drugs of interest (\%)

\begin{tabular}{|c|c|c|c|c|c|c|c|c|c|c|c|}
\hline SLE & 2006 & 2007 & 2008 & 2009 & 2010 & 2011 & 2012 & 2013 & 2014 & 2015 & 2016 \\
\hline Prednisone & 40.0 & 35.9 & 33.6 & 32.9 & 32.0 & 34.4 & 33.4 & 33.5 & 32.0 & 32.6 & 34.7 \\
\hline Methylprednisolone & 18.7 & 19.4 & 18.7 & 21.3 & 21.7 & 19.7 & 21.2 & 22.0 & 22.0 & 22.9 & 24.4 \\
\hline Other Steroids & 13.7 & 14.1 & 13.9 & 14.3 & 15.7 & 15.6 & 16.1 & 16.6 & 16.8 & 17.1 & 19.7 \\
\hline Anti-Malarials & 50.1 & 46.4 & 44.7 & 44.8 & 45.4 & 46.4 & 46.8 & 47.2 & 46.1 & 45.1 & 47.5 \\
\hline Methotrexate & 9.1 & 8.1 & 8.1 & 7.5 & 8.1 & 7.9 & 7.9 & 8.1 & 8.1 & 7.7 & 8.1 \\
\hline Leflunomide & 1.4 & 1.4 & 1.5 & 1.4 & 1.3 & 1.3 & 1.4 & 1.6 & 1.6 & 1.6 & 1.9 \\
\hline Azathioprine & 6.6 & 5.6 & 4.9 & 4.8 & 4.5 & 4.5 & 4.2 & 4.3 & 4.3 & 4.3 & 4.8 \\
\hline Mycophenolate & 6.3 & 4.9 & 4.6 & 4.5 & 4.5 & 4.5 & 4.5 & 4.8 & 4.4 & 4.6 & 5.0 \\
\hline Cyclophosphamide & 1.2 & 1.0 & 0.9 & 0.9 & 0.8 & 0.5 & 0.5 & 0.6 & 0.4 & 0.3 & 0.3 \\
\hline Tacrolimus & 0.4 & 0.4 & 0.4 & 0.4 & 0.4 & 0.4 & 0.5 & 0.4 & 0.4 & 0.5 & 0.6 \\
\hline Cyclosporine & 0.7 & 0.5 & 0.4 & 0.4 & 0.4 & 0.3 & 0.3 & 0.3 & 0.2 & 0.2 & 0.2 \\
\hline Belimumab & 0.0 & 0.0 & 0.0 & 0.0 & 0.0 & 0.3 & 0.8 & 1.0 & 1.1 & 1.2 & 1.4 \\
\hline Rituximab & 0.6 & 0.7 & 0.7 & 0.7 & 0.7 & 0.7 & 0.7 & 0.8 & 0.8 & 0.8 & 1.0 \\
\hline No Medication & 23.7 & 26.9 & 29.6 & 28.3 & 28.2 & 28.0 & 27.6 & 26.7 & 27.9 & 28.3 & 24.7 \\
\hline $\mathbf{L N}$ & 2006 & 2007 & 2008 & 2009 & 2010 & 2011 & 2012 & 2013 & 2014 & 2015 & 2016 \\
\hline Prednisone & 49.4 & 44.6 & 44.9 & 44.6 & 41.5 & 44.1 & 44.0 & 42.9 & 39.6 & 40.7 & 41.9 \\
\hline Methylprednisolone & 13.1 & 15.3 & 16.0 & 19.6 & 17.9 & 18.2 & 19.7 & 20.6 & 20.7 & 20.7 & 22.2 \\
\hline Other Steroids & 10.0 & 11.1 & 11.6 & 14.9 & 15.8 & 14.7 & 14.1 & 17.1 & 16.3 & 16.6 & 17.9 \\
\hline Anti-Malarials & 38.8 & 39.3 & 41.2 & 41.3 & 41.7 & 43.9 & 44.7 & 46.2 & 42.5 & 42.7 & 45.1 \\
\hline Methotrexate & 2.7 & 3.9 & 4.6 & 5.2 & 6.5 & 5.2 & 5.7 & 6.4 & 6.0 & 5.6 & 5.4 \\
\hline Leflunomide & 1.5 & 1.6 & 1.9 & 1.9 & 1.6 & 1.6 & 1.7 & 1.7 & 1.6 & 1.3 & 1.9 \\
\hline Azathioprine & 6.4 & 6.2 & 6.1 & 6.2 & 6.0 & 6.1 & 6.0 & 5.7 & 5.5 & 5.4 & 5.4 \\
\hline Mycophenolate & 19.1 & 15.2 & 14.7 & 14.2 & 14.5 & 13.9 & 14.1 & 14.5 & 13.4 & 14.0 & 14.8 \\
\hline Cyclophosphamide & 4.2 & 2.3 & 2.2 & 2.1 & 2.6 & 1.6 & 2.0 & 2.3 & 1.6 & 1.1 & 1.3 \\
\hline Tacrolimus & 2.5 & 2.2 & 2.2 & 2.3 & 2.2 & 2.2 & 2.3 & 2.0 & 1.6 & 1.9 & 2.3 \\
\hline Cyclosporine & 2.6 & 1.8 & 1.9 & 2.0 & 1.7 & 1.6 & 1.2 & 1.1 & 0.7 & 0.7 & 0.6 \\
\hline Belimumab & 0.0 & 0.0 & 0.0 & 0.0 & 0.0 & 0.4 & 1.1 & 1.3 & 1.2 & 1.1 & 1.4 \\
\hline Rituximab & 0.8 & 1.1 & 0.6 & 1.1 & 1.0 & 1.0 & 1.0 & 1.3 & 1.2 & 1.2 & 1.6 \\
\hline No Medication & 24.0 & 25.9 & 24.0 & 22.7 & 24.9 & 25.2 & 22.1 & 22.8 & 25.4 & 25.0 & 24.2 \\
\hline
\end{tabular}

